\title{
Boundary effects in a surface reaction model for $\mathrm{CO}$ oxidation
}

\author{
Benjamin J. Brosilow, Erdogan Gulari, and Robert M. Ziff \\ Department of Chemical Engineering, The University of Michigan, Ann Arbor, Michigan 48109-2136
}

(Received 4 September 1992; accepted 22 September 1992)

The surface reaction model of Ziff, Gulari, and Barshad (ZGB) is investigated on finite systems with "hard" oxygen boundary conditions. The rate of production of $\mathrm{CO}_{2}$ is calculated as a function of $y$ and system size. When the rate of $\mathrm{CO}$ adsorption $y$ is above the firstorder transition value $y_{2}$, the reactive region is found to extend into the system a distance $\xi$ which scales as $\left(y-y_{2}\right)^{-0.40}$ when $y \rightarrow y_{2}$.

\section{INTRODUCTION}

The $A-B_{2}$ reaction model of Ziff, Gulari, and Barshad (ZGB) ${ }^{1}$ is a simple stochastic (Monte Carlo) model for the reaction $\mathrm{CO}+\frac{1}{2} \mathrm{O}_{2} \rightarrow \mathrm{CO}_{2}$ catalyzed by a single crystal of platinum. The model has allracted attention due to its ability to describe some of the anomalous features of the $\mathrm{CO}$ oxidation reaction, and also due to the complex dynamic behavior that arises from its simple formulation. ${ }^{2-10}$ In this note, we present the results of an investigation of the model's behavior in finite and semi-infinite systems with hard (nonperiodic) boundary conditions. Our motivation for performing this study stems from recent experimental investigations of low temperature $\mathrm{CO}$ oxidation over supported catalysts in which the platinum is dispersed as isolated islands over an oxide support. ${ }^{11-14}$

\section{THE ZGB MODEL}

A detailed description of the ZGB model can be found in Ref. 1 , so we present only a brief description here. The model incorporates the following steps: adsorption of $\mathrm{CO}$ molecules onto single empty sites, dissociative adsorption of $\mathrm{O}_{2}$ molecules onto pairs of adjacent empty sites, and reaction of $\mathrm{CO}^{*}-\mathrm{O}^{*}$ nearest neighbor pairs. (The asterisk indicates an adsorbed species.) In the model's simplest form, nonreactive desorption and surface diffusion are ignored. Letting $S$ denote a vacant site, we can summarize steps of the model by

$$
\begin{array}{ll}
\mathrm{CO}+S \rightarrow \mathrm{CO}^{*} & \text { rate } \propto y, \\
\mathrm{O}_{2}+2 S \rightarrow 2 \mathrm{O}^{*} & \text { rate } \propto(1-y), \\
\mathrm{CO}^{*}+\mathrm{O}^{*} \rightarrow 2 S+\mathrm{CO}_{2} \uparrow & \text { rate }=\infty \text { (for nearest neighbors). }
\end{array}
$$

Simulations on large lattices with periodic boundary conditions have shown that for $y<y_{1} \approx 0.387$ (Refs. 1517) the system eventually saturates with $O^{*}$, while for $y>y_{2} \approx 0.525589$ (see below), the system eventually saturates with $\mathrm{CO}^{*}$. For $y_{1}<y<y_{2}$ the system reaches a steady state where reaction continues (nearly) indefinitely. In this reactive state, there are both $\mathrm{CO}^{*}$ and $\mathrm{O}^{*}$ sites on the lattice, separated by unoccupied sites where further adsorption and reaction can occur. The transition to the $\mathrm{CO}^{*}$ saturated state at $y_{2}$ is first order, as the fractional coverage of each surface species is discontinuous in $y$ at this transition. The transition from the $\mathrm{O}^{*}$ saturated state at $y_{1}$ is second order, as the surface coverages are continuous in $y$, while the derivative of the coverages are discontinuous in $y$ at this transition.

\section{MOTIVATION FOR INVESTIGATING THE MODEL ON FINITE SYSTEMS}

The ZGB model was originally developed as a model for $\mathrm{CO}$ oxidation over a macroscopic single crystal of platinum. The model's lattice was intended to represent a face of a large platinum single crystal, with each lattice site corresponding to one surface platinum atom. With this physical system in mind, most studies have concentrated on investigation of the model's behavior on large lattices with periodic boundaries. Restricting study to such lattices also simplifies investigation of the model by eliminating the need for detailed consideration of the reaction dynamics near the lattice boundary.

The present investigation of this model on finite systems was motivated by recent experimental studies of the $\mathrm{CO}$ oxidation reaction over noble metal/reducible oxide catalysts. These catalysts have attracted some attention due to their ability to catalyze the $\mathrm{CO}$ oxidation reaction at temperaturcs as low as $-70^{\circ} \mathrm{C} .{ }^{11-14}$ Some examples of these catalysts are $\mathrm{Au}$ or $\mathrm{Pt}$ on $\mathrm{MnO}_{x}$ or $\mathrm{Fe}_{2} \mathrm{O}_{3}$. The surfaces of these catalysts are composed mostly of the oxide, with the noble metal being present in the form of islands that are typically on the order of tens or hundreds of angstroms in diameter. ${ }^{11-14}$ (The method of catalyst preparation determines the average size of the metal islands). The precise mechanism by which the $\mathrm{CO}$ oxidation proceeds over these catalysts is not known; however, it is believed that the reaction is localized around the noble metal islands, since the pure oxides do not catalyze the reaction appreciably. ${ }^{11,12}$ Also, CO oxidation typically occurs via a Langmuir-Hinshelwood mechanism on large Pt or Pd crystals. ${ }^{18,19}$ It is believed that the reducible oxide support can adsorb oxygen from the gas phase, but will not adsorb $\mathrm{CO}^{20}$

Since relatively little is known about the mechanism of the oxidation reaction on noble metal/reducible oxide catalysts, we have chosen to investigate one particularly simple possible mechanism. We assume that the reaction occurs only on the metal islands, and that it proceeds by a Langmuir-Hinshelwood mechanism (i.e., both reactants must adsorb on the catalyst before they can react). The oxide serves as an infinite reservoir of oxygen surrounding 
the metal island. Thus our model consists of a finite lattice on which we run the ZGB model, with the sites along the perimeter of the lattice constantly maintained in an $\mathrm{O}^{*}$ state.

\section{THE MODEL ON FINITE SYSTEMS}

The first system that we investigated was an $L \times L$ square lattice on which all sites inside and on a circle of radius $R<L / 2$ about the central lattice point were initially unoccupied, and all sites outside this circle were initially occupied by $\mathrm{O}^{*}$. The standard ZGB model (as described in Ref. 1) was then run on the lattice, except that whenever an oxygen atom on a site outside the circle of radius $R$ reacted with a $\mathrm{CO}^{*}$ molecule from inside the circle, the resulting unoccupied site outside the circle was immediately returned to an $\mathrm{O}^{*}$ state. Thus the sites outside the circle were always in the $O^{*}$ state. Figures 1 (a) and 1(b) show typical states for a system with $R=31$. We ran computer simulations of such systems for various $R$ and $y$, and in Fig. 2 we plot the rate of production of $\mathrm{CO}_{2}$ per catalyst site as a function of $y$ for various $R$. (Here the number of catalyst sites is taken to be the number of lattice sites inside and on the circle of radius $R$.) Note that the rate of production of $\mathrm{CO}_{2}$ per catalyst site is $r_{\mathrm{CO}_{2}}=y \theta_{S}$, where $\theta_{S}$ is the time averaged fraction of catalyst sites that are unoccupied. This relationship follows from the following observation: The probability of successfully adsorbing a $\mathrm{CO}$ molecule is $y \theta_{S}$, and at steady state, every $\mathrm{CO}$ adsorption must be matched by a $\mathrm{CO}_{2}$ desorption in order to prevent accumulation of $\mathrm{CO}^{*}$ on the lattice. Figure 2 also shows the previously known result for the reaction rate per catalyst site on an infinite system (i.e., $R \rightarrow \infty$ ). ${ }^{1}$

Figure 2 shows that within the region $y_{1}<y<y_{2}$ the system with $R=127$ shows behavior similar to that of an infinite system, with a sharp drop in reaction rate near $y_{2}$. (Note that there is presumably no discontinuity in the rate of reaction for a finite system, so we define the first-order transition point in our finite systems as the point at which the reaction rate reaches a maximum.) For $y>y_{2}$ the $R=127$ system maintains a small and nearly constant reaction rate. In this region, the catalyst is almost entirely covered by $\mathrm{CO}^{*}$ inside the circle of radius $R$, except very near the perimeter of the circle, where the reaction continues to occur [somewhat like the situation shown in Fig. 1 (b)]. For systems with smaller $R$, Fig. 2 shows that the region corresponding to the reactive region for an infinite system $\left(y_{1}<y<y_{2}\right)$ seems to be translated to slightly higher values of $y$, and the drop in the reaction rate to the right of the first-order transition point becomes smaller and more gradual.

We did not investigate the behavior of these systems close to the infinite-system second-order transition point $y_{1}$, because for $y$ close to $y_{1}$ the finite systems tended to fluctuate into the stationary, nonreactive state where all the lattice sites were occupied by $\mathrm{O}^{*}$. We note that all the steady states shown in Fig. 2 are technically only metastable states, since any finite system with $y<1$ will presumably fluctuate into a stationary, nonreactive $\mathrm{O}^{*}$-saturated
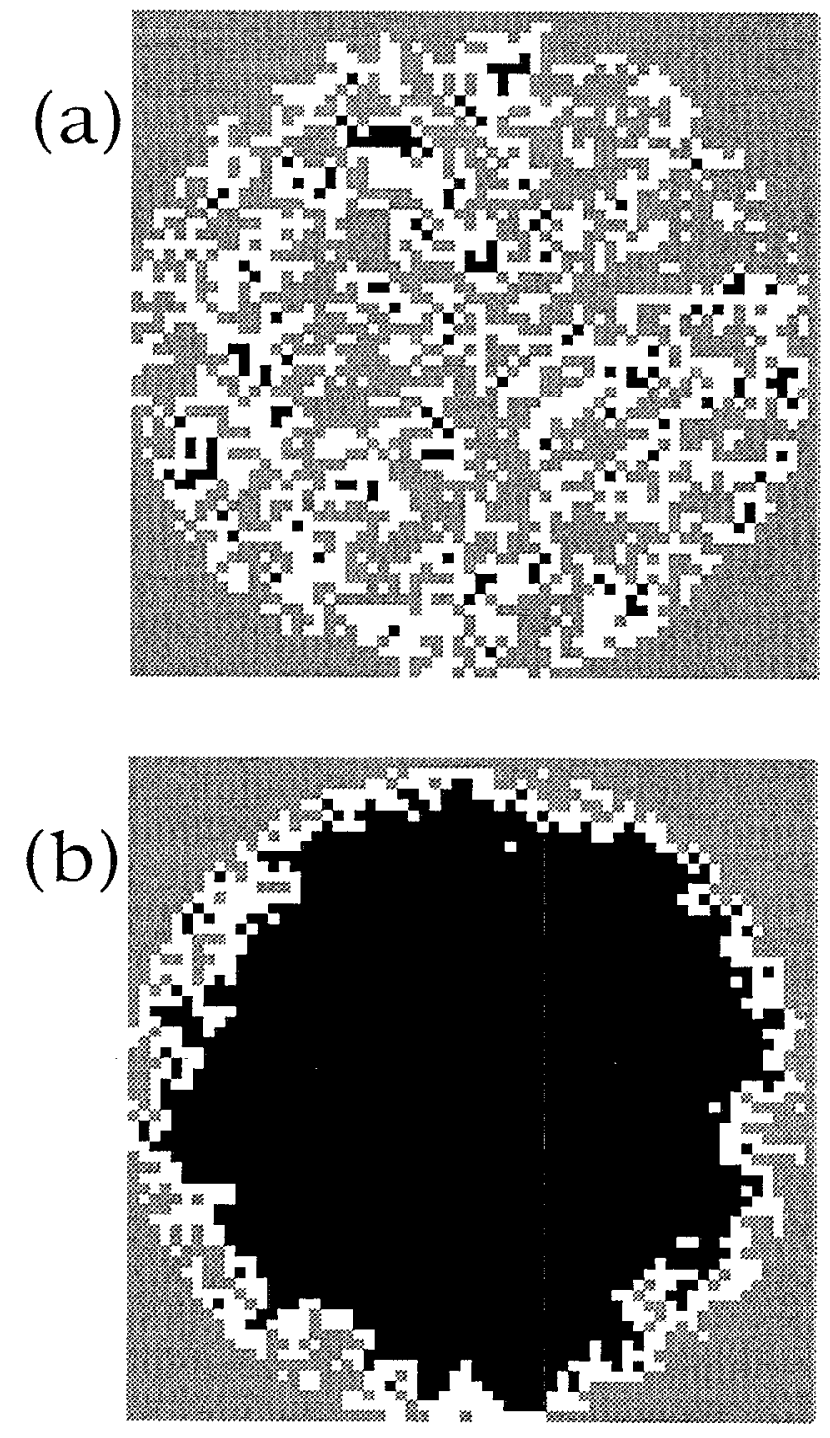

FIG. 1. Typical lattice configurations for the finite system model with $R=31$ at (a) $y=0.5255$ (just below the transition at $y_{2}=0.525589$ ), and (b) $y=0.54$ (above the transition). Adsorbed $\mathrm{CO}^{*}$ are shown as black dots, adsorbed $\mathrm{O}^{*}$ are shown as grey, and unoccupied lattice sites are white.

state after a finite amount of time. However, each simulation used to generate Fig. 2 was run for over $1 \times 10^{7}$ Monte Carlo steps (MCS), and such nonreactive states were never reached.

\section{THE MODEL ON SEMI-INFINITE SYSTEMS}

As shown in Fig. 1(b), values of $y$ above the first-order transition value result in a lattice where the central region is saturated with $\mathrm{CO}^{*}$, and the reaction proceeds only within an annulus around the edge. The next series of simulations which we ran were directed at characterizing the width of this reactive annulus as a function of $y$. We ran these simulations on a lattice of $L \times W$ sites, where the left-most column of $W$ sites was initially occupied by $\mathrm{O}^{*}$, the right-most $L / 2$ columns were initially occupied by $\mathrm{CO}^{*}$, and the remaining sites were initially vacant. The 


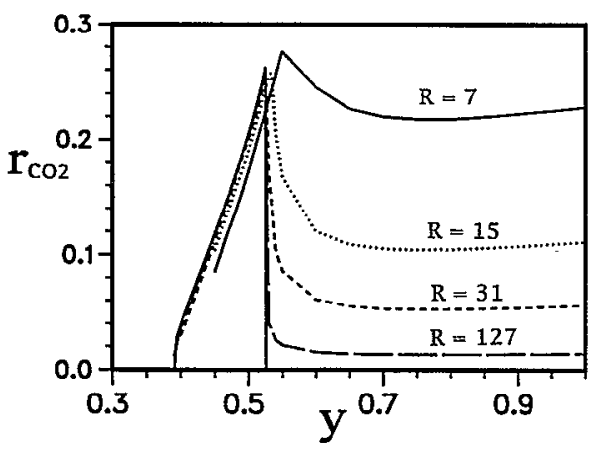

FIG. 2. The rate of production of $\mathrm{CO}_{2}$ per catalyst site per MCS as a function of $y$ for systems with various $R$. The unlabeled solid curve shows the rate of production of $\mathrm{CO}_{2}$ on an infinite system $(R \rightarrow \infty)$.

standard ZGB model was run on this lattice, except that whenever an $\mathrm{O}^{*}$ was removed (through reaction) from the left-most column of the lattice, it was immediately replaced by another $\mathrm{O}^{*}$. In this way, all the sites in the left-most column were always in the $\mathrm{O}^{*}$ state. Periodic boundary conditions were used in the vertical direction (as oriented in Fig. 3 ) and the length $L$ of the system was chosen large enough so that the right-most column of $\mathrm{CO}^{*}$ did not react and desorb from the surface for the duration of the simulation, and hence the particular rules for reaction along this boundary need not be specified. Figure 3 shows a typical state of the lattice in one of these simulations.

We ran these simulations at various values of $y>y_{2}$, and recorded the average local concentration of the various surface species as a function of distance from the $\mathrm{O}^{*}$ boundary at the left of the lattice. In Fig. 4 we show a profile of these concentrations for $y=0.52563$. Figure 5 shows the local rate of production of $\mathrm{CO}_{2}$ as a function of distance from the $\mathrm{O}^{*}$ boundary for various values of $y$. The

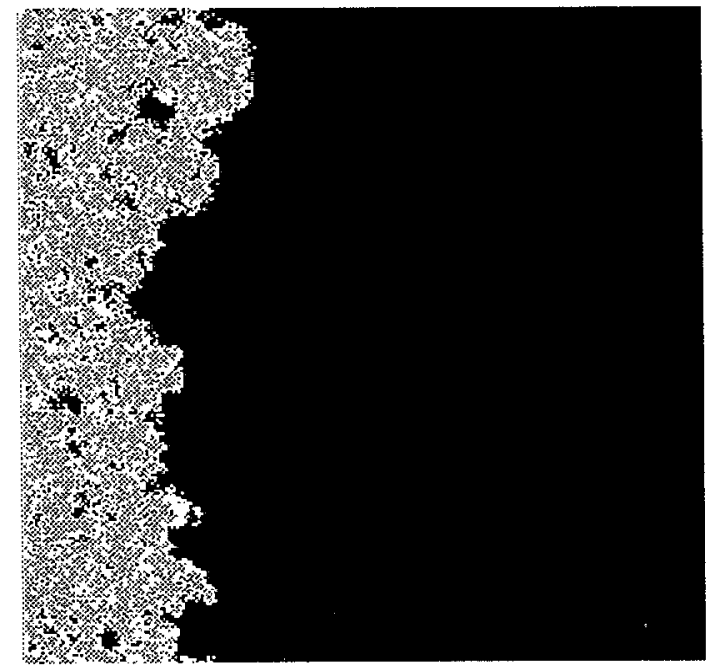

FIG. 3. Typical lattice configuration for the model on a semi-infinite lattice with $y=0.52563$. Adsorbed $\mathrm{CO}^{*}$ are shown as black dots, adsorbed $\mathrm{O}^{*}$ are shown as grey, and unoccupied lattice sites are white.

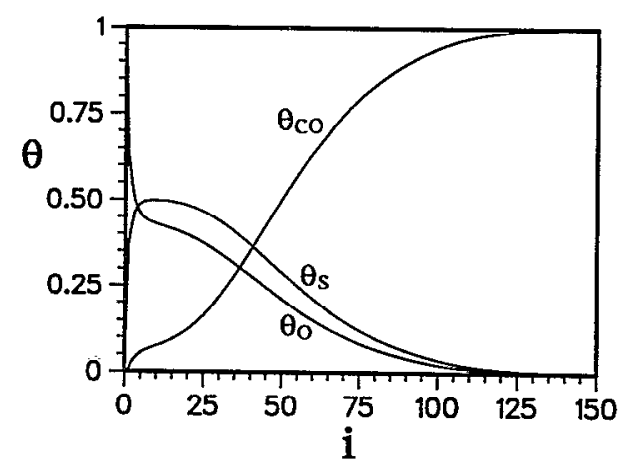

FIG. 4. Concentration profile for the various surface species as a function of distance from the oxygen boundary, for the semi-infinite system with $y=0.52563 . i$ is perpendicular distance from the $\mathrm{O}^{*}$ boundary measured in lattice sites. $\theta_{X}$ denotes the fractional coverage of species $X$.

rate of reaction in column $i$ was calculated as $r_{i}=y \theta_{S, i}$, where $\theta_{S, i}$ is the time-averaged fraction of vacant sites in column $i$ of the lattice. If we define the width of the reactive strip for these systems as $\xi=\Sigma i r_{i} / \Sigma r_{i}$, then the plot in Fig. 6 shows that this width scales with $y$ as $\xi=k(y$ $\left.-y_{2}\right)^{-v}$, with $v=0.40$ and $k=0.74$. To our knowledge, this is the first scaling relation about $y_{2}$ that deals with spatial correlations for this model. (Evans and Miesch ${ }^{4}$ have previously investigated scaling relations about $y_{2}$ that deal with temporal correlations.) The lattice dimensions in the simulations described in this section were $L=W=256$ sites, and the simulations were initialized for $1 \times 10^{6} \mathrm{MCS}$ before data collection was started, except for the systems with $y-y_{2}<0.003$, which were initialized for over $1 \times 10^{7}$ MCS. Data collection was carried out for between $2 \times 10^{4}$ and $1 \times 10^{8} \mathrm{MCS}$, depending on the value of $y-y_{2}$.

In order to accurately determine how $\xi$ scaled with $y-y_{2}$, it was necessary to determine the value of $y_{2}$ to greater accuracy than was previously known. For this purpose we ran a "constant-coverage" simulation to determine $y_{2}{ }^{21}$ Our simulation was similar to that described in Ref. 21 , except that we used a system of $256 \times 512$ sites at a coverage $\theta_{A}=0.40$, and we used a greater degree of optimization in the computer code. The simulation was run for over $3.85 \times 10^{7}$ MCS (after an initialization period of 1

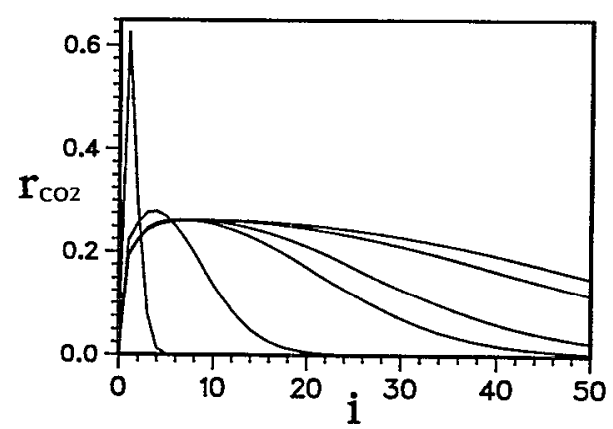

FIG. 5. The localized rate of reaction in column $i$ of the semi-infinite system for various values of $y$. The curves are, from left to right: $y=0.7$, $0.53,0.526,0.5258,0.52565$, and 0.52563 . 


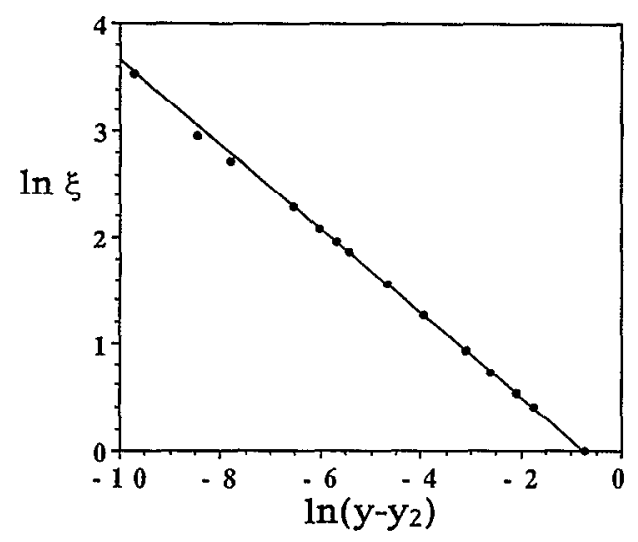

FIG. 6. A $\ln -\ln$ plot of $\xi$, the width of the reactive region in the semiinfinite system, vs $\left(y-y_{2}\right)$. The line satisfies the equation $\ln (\xi)=-0.30$ $-0.40 \ln \left(y-y_{2}\right)$.

$\left.\times 10^{6} \mathrm{MCS}\right)$, and we determined $y_{2}=0.525589$ \pm 0.000002 , where the uncertainty represents one standard deviation as described in Ref. 21 . Our result is in agreement with, but morc precise than the valuc reported in Ref. 21, and this new value was used in plotting the data of Fig. 6. The use in Fig. 6 of a value for $y_{2}$ that deviated from the above value by more than two standard deviations results in the data showing noticeable curvature.

\section{CONCLUSIONS}

We have investigated the behavior of the ZGB model on finite and semi-infinite systems. For finite circular systems with $y<y_{2}$ we found that the behavior of the model was similar to its behavior on infinite systems. Finite systems with sufficiently large $R$ ( $R \gtrsim 31$ sites) showed firstorder transitionlike behavior near $y=y_{2}$, and systems with smaller $R$ showed a morc gradual dccreasc and leveling off of the reaction rate. For $y \gtrsim 0.7$, the finite systems showed a rate of production of $\mathrm{CO}_{2}$ that was virtually independent of $y$. On a semi-infinite square lattice with one boundary maintained in the $\mathrm{O}^{*}$ state, we found the scaling relation $\xi=k\left(y-y_{2}\right)^{-0.40}$, where $\xi$ is the average depth of penetration of the reaction into the $\mathrm{CO}^{*}$ saturated region of the lattice. We believe this to be the first investigation of a scaling relation about $y_{2}$ dealing with spatial correlations in the model.

\section{ACKNOWLEDGMENT}

The authors acknowledge support from the U. S. National Science Foundation, Grant No. DMR 9122341.

${ }^{1}$ R. M. Ziff, E. Gulari, and Y. Barshad, Phys. Rev. Lett. 56, 2553 (1986).

${ }^{2}$ M. Ehsasi, M. Matloch, O. Frank, J. H. Block, K. Christmann, F. S. Rys, and W. Hirschwald, J. Chem. Phys. 91, 4949 (1989).

${ }^{3}$ E. V. Albano, J. Chem. Phys. 94, 1499 (1991).

${ }^{4}$ J. W. Evans and M. S. Miesch, Phys. Rev. Lett. 66, 833 (1991).

${ }^{5}$ B. Yu, D. A. Browne, and P. Kleban, Phys. Rev. A 43, 1770 (1991).

${ }^{6}$ J. Mai and W. von Niessen, Phys. Rev. A 44, R6165 (1991).

${ }^{7}$ A. Yu. Tretyakov and H. Takayasu, Phys. Rev. A 44, 8388 (1991).

${ }^{8}$ D. ben-Avraham, S. Redner, D. B. Considine, and P. Meakin, J. Phys. A 23, L613 (1990).

${ }^{9}$ M. Kolb and Y. Boudeville, J. Chem. Phys. 92, 3935 (1990).

${ }^{10}$ J. J. Luque, Phys. Rev. A 42, 3319 (1990).

${ }^{11}$ M. Haruta, T. Kobayashi, H. Sano, and N. Yamada, Chem. Lett., 405 (1987).

${ }^{12}$ M. Huruta, N. Yamada, T. Kobayashi, and S. Iijima, J. Catal. 115, 301 (1989).

${ }^{13}$ S. D. Gardner, G. B. Hoflund, D. R. Schryer, J. Schryer, B. T. Upchurch, and E. J. Kielin, Langmuir 7, 2135 (1991).

${ }^{14}$ S. D. Gardner, G. B. Hollund, M. R. Davidson, H. A. Laitinen, D. R. Schryer, and B. T. Upchurch, Langmuir 7, 2140 (1991).

${ }^{15}$ I. Jensen, H. C. Fogedby, and R. Dickman, Phys. Rev. A 41, 3411 (1990).

${ }^{16}$ G. Grinstein, Z. W. Lai, and D. A. Browne, Phys. Rev. A 40, 4820 (1989).

${ }^{17}$ P. Meakin and D. J. Scalapino, J. Chem. Phys. 87, 731 (1987).

${ }^{18}$ T. Engel and G. Ertyl, J. Chem. Phys. 69, 1267 (1978).

${ }^{19}$ T. Engel and G. Ertyl, Adv. Catal. 28, 1 (1979).

${ }^{20}$ B. C. Gates, J. R. Katzer, and G. C. A. Schuit, Chemistry of Catalytic Process (McGraw-Hill, New York, 1979).

${ }^{21}$ Ziff and Brosilow (to be published). 